\title{
Fisioterapia en Animales Domésticos ¿Qué dice la evidencia?
}

José Iván Alfonso Mantilla Jaime Martínez Santa

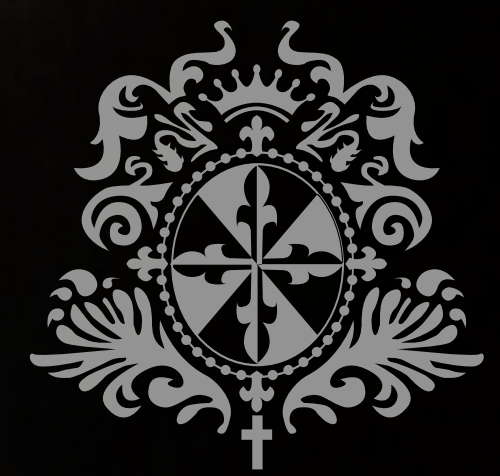


Title: $\quad$ Physiotherapy in Domestic Animal

Subtitle: What does the evidence say?

Título: $\quad$ Fisioterapia en Animales Doméstic

Subtítulo: ¿Qué dice la evidencia?

Alt title / Título Alternativo:

[en] Physiotherapy in Domestic Animal What does the evidence say?

[es] Fisioterapia en Animales Domésticos: ¿Qué dice la evidenci

Author (s) / Autor (es):

Alfonso Mantilla \& Martínez Santa

Keywords / Palabras Clave:

[en] dogs; cats; veterinary medicine; rehabilitation animal's

[es] perros; gatos; medicina veterinaric rehabilitación de animales

Submited: 2017-05-20

\section{Resumen}

Introducción: la medicina veterinaria, es un campo de conocimiento que integra diversas áreas profesionales para su desarrollo, es así como se ha reportado evidencia en relación a la participación de fisioterapeutas en procesos de rehabilitación de animales que presentan algún tipo de patología que afecte su funcionalidad. Objetivo: realizar una revisión de la literatura con relación a las intervenciones fisioterapéuticas realizadas en perros y gatos con alteraciones osteomusculares y neuromusculares. Materiales y métodos: Se realizó una revisión de la literatura, búsqueda en bases de datos como: Ebsco, Pedro, Hinari, Elsevier, Science Direct, Springer y Medline que contemplaran los siguientes criterios de inclusión: estudios publicados del año 2000 al 2016, literatura con

los siguientes términos DeCS: dogs, cats, veterinary medicine, rehabilitation, animals. Resultados: se obtuvo información en relación a la intervención fisioterapéutica

en perros y gatos, se ha reportado el uso de agentes

físicos para el tratamiento de lesiones agudas a través

de modalidades como crioterapia, termoterapia,

ultrasonido y electroestimulación; el uso de estos agentes físicos ayuda al manejo de dolor, disminución del edema, aumento del rango movimiento y reducción

de la espasticidad, además de eso se reporta que el ejercicio terapéutico y terapia manual tienen efectos significativos en el retorno a las actividades de la vida diaria de estos animales. Conclusiones: la fisioterapia en animales está indicada para procesos agudos y crónicos

como lesiones osteomusculares y neuromusculares,

esta se realiza a través de medios físicos y ejercicio terapéutico enfocándose en actividades funcionales de movimiento.

\section{Abstract}

Background: Veterinary medicine is a field of knowledge that integrates several professional areas for its

development. This is how evidence has been reported regarding the participation of physiotherapists in the rehabilitation processes of animals that present some type of pathology that affects its functionality. Objective: perform a review of the literature regarding the physiotherapeutic interventions performed

in dogs and cats with musculoskeletal and neuromuscular disorders. Materials and methods: A review of the literature,

search in databases such as "Ebsco"

"Pedro" "Hinari" "Elsevier" "Science

Direct" "Springer" "Medline", which

included the following inclusion criteria: 2000 to 2016, literature with the following terms DeCs: dogs, cats, veterinary medicine, rehabilitation, animals.

Results: We found information in which information was obtained regarding the physiotherapeutic intervention in dogs and cats, it has been reported the use

of physical agents for the treatment of acute injuries through modalities such as cryotherapy, thermotherapy, ultrasound and electrostimulation, the use of these

physical agents helps to manage pain, decrease edema, increase range of motion

and reduce spasticity, in addition to that it is reported that the therapeutic exercise and manual therapy have significant effects on the return to activities of daily life of These animals. Conclusions: Physiotherapy in animals is indicated for acute and chronic processes such as musculoskeletal and neuromuscular lesions, this is done through physical means and therapeutic exercise focusing on functional activities of movement.

Acepted: 2017-06-20

\author{
José Iván Alfonso Mantilla, Pt \\ ResearchID: $\quad$-4973-2017 \\ OrclD: $\quad$ 0000-0003-2597-1826 \\ Bio: \\ Fisioterapeuta, Universidad del Rosario \\ Filiación: \\ Universidad del Rosario \\ Ciudad: \\ Bogotá D.C. [co] \\ e-mail: \\ josealfonso25@hotmail.com
}

\author{
Jaime Martínez Santa, Pt sp \\ Bio: \\ Fisioterapeuta, Universidad Nacional de Colombia \\ Especialista en Epidemiología, Universidad del Rosario \\ Especialista en Estadística, Universidad Nacional de Colombia \\ Especialista en Antropología Forense, Universidad Nacional de Colombia \\ Especialista en Fisioterapia en el Adulto Crítico, Universidad del Rosario \\ Ciudad: \\ Bogotá D.C. [co] \\ e-mail: \\ jmartinezsanta@yahoo.com
}




\section{Fisioterapia en Animales Domésticos ¿Qué dice la evidencia?}

Physiotherapy in Domestic Animals: What does the evidence say?

\section{José Iván Alfonso Mantilla Jaime Martínez Santa}

En la actualidad la rehabilitación en animales se encuentra como una de las líneas de investigación en medicina veterinaria. Cirujanos, internistas, neurólogos y fisioterapeutas han reportado literatura sobre la rehabilitación animal, abriendo una puerta a este nuevo campo de investigación en el mundo. (Drum, 2010; Hanks, Levine, \& Bockstahler, 2015; Challande Kathmann, Demierre, \& Jaggy, 2001; Michel \& Brown, 2011; Millis \& Ciuperca, 2015; Nolen, 2009)

En primera instancia, en el Reino Unido se realizan procesos de rehabilitación en perros y gatos basados en intervenciones fisioterapéuticas en lesiones osteomusculares y neuromusculares basadas en ejercicio terapéutico y agentes físicos. Además, la práctica de la fisioterapia en animales se realiza bajo la dirección de un médico veterinario. (Henderson, Latimer, \& Millis, 2015)

\section{Rehabilitación y recuperación}

En medicina se ha determinado que la rehabilitación es un pilar en los procesos de recuperación de pacientes con diferentes tipos de patología. De forma similar, en medicina veterinaria la rehabilitación es un paso importante en la recuperación de animales con patologías osteomusculares y neuromusculares. Entre las lesiones más comunes en animales, como perros y gatos, se encuentran las fracturas articulares y peri articulares, fracturas femorales distales y proximales, fracturas humerales, tendosinovitis bicipitales, inestabilidad escapular, miopatía fibrótica, alteraciones musculares y lesiones de tendón. (Henderson, Latimer, \& Millis, 2015)

Según la fundación ortopédica para animales, la presencia de displasia de cadera en perros es de $21 \%$ sobre la base de 430.000 evaluaciones realizadas y la displasia de codo está presente en el 16\% basado en 180.000 evaluaciones realizadas. (Marcellin Little, Doyle, \& Pyke, 2015; Sims, Waldron, \& Marcellin Little, 2015). Desde rehabilitación los objetivos principales son el manejo de dolor, la restauración de la función perdida en el animal y el retorno a actividades de la vida diaria. (Marcellin Little, Doyle, \& Pyke, Physical rehabilitation after total joint arthroplasty in companion animals, 2015)
Se ha reportado que existen perros y gatos con enfermedades neurológicas, estas comprometen la capacidad de movilización de extremidades, micción y cambios de posición de decúbito lateral a una posición esternal. La rehabilitación puede durar desde semanas a meses, el principal enfoque es el retorno a actividades de la vida diaria y la preservación de la fuerza muscular. (Marcellin Little, Doyle, \& Pyke, Physical rehabilitation after total joint arthroplasty in companion animals, 2015)

Dentro de las acciones fisioterapéuticas se encuentra la creación de prótesis y órtesis las cuales permiten el desarrollo del patrón de locomoción, inmovilización y limitación de rango de movimiento para garantizar procesos de cicatrización. En este proceso, el profesional en rehabilitación actúa en la adaptación de estos equipos en perros y gatos. (Marcellin Little, Drum, Levine, \& McDonald, 2015)

En la última década, la fisioterapia ha tomado un papel fundamental dentro de los procesos de rehabilitación en medicina veterinaria. Por ejemplo, dentro de las estrategias terapéuticas utilizadas está el uso de agentes físicos, ejercicio terapéutico, prescripción de órtesis y prótesis y terapia manual. Dentro de los objetivos de la rehabilitación se encuentra el aumento del rango de movimiento, incremento de la independencia funcional del paciente veterinario después de un proceso quirúrgico, y alivio de dolor. (Drum, Bockstahler, Levine, \& Marcellin Little, 2015; Levine, Marcellin Little, Millis, Tragauer, \& Osborne, 2010) 


\section{Fisioterapia en Animales Domésticos}

¿Qué dice la evidencia

En el mundo, perros y gatos padecen de enfermedades osteomusculares y neuromusculares que deben ser tratadas por profesionales en rehabilitación con herramientas de intervención específicas. El fisioterapeuta es un profesional encargado de analizar e intervenir patologías que afecten el movimiento corporal humano. De hecho, el fisioterapeuta puede intervenir alteraciones del movimiento en animales debido a sus bases teóricas en el área de rehabilitación. Es por eso, el fisioterapeuta debe ser parte del equipo interdisciplinar de medicina veterinaria para la atención de este tipo de población. El objetivo de este artículo es realizar una revisión de la literatura sobre las intervenciones fisioterapéuticas realizadas en perros y gatos con alteraciones osteomusculares y neuromusculares.

\section{Materiales y métodos}

Se realizó una revisión de la literatura en bases de datos como: Ebsco, Pedro, Hinari, Elsevier, Science Direct, Springer, Medline; para la selección de recursos se determinaron los siguientes criterios:

\section{Criterios de Inclusión}

Estudios publicados del año 2000 al 2016, literatura con los siguientes términos DeCS: dogs, cats veterinary medicine, rehabilitation, animals.

Se estableció como otro criterio de inclusión que la búsqueda de evidencia se basara en: Fuentes primarias: estudios ECA, Metaanálisis, casos y controles, estudios de cohorte, revisiones sistemáticas, revisiones de literatura. Fuentes secundarias: Monografías, tesis de grado, libros, además que la población de la evidencia fueran animales que hubieran tenido contacto con fisioterapia o rehabilitación, idioma de la evidencia español, inglés o portugués.

\section{Criterios de exclusión}

Estudios que su año de publicación fuera previo al año 2000 o que no contemplaran los términos DeCS establecidos, estudios que no estuvieran disponibles o completos.

\section{Criterio de calidad}

La calidad de los artículos incluidos en la revisión, se realizó a través de los criterios de chequeo dados por la Scottish Intercollegiate Guidelines Network - SIGN, estas listas incluyen elementos de evaluación tales como: pertinencia, validez interna y calidad de los estudios incluidos en la revisión.

\section{Resultados}

El Gráfico 1 ilustra el proceso seguido para la extracción de la evidencia; en la Tabla 1 se muestran los tipos de estudio analizados en este trabajo. Al final de ese apartado, la Tabla 2 resume las principales patologías en población veterinaria y las intervenciones fisioterapéuticas.

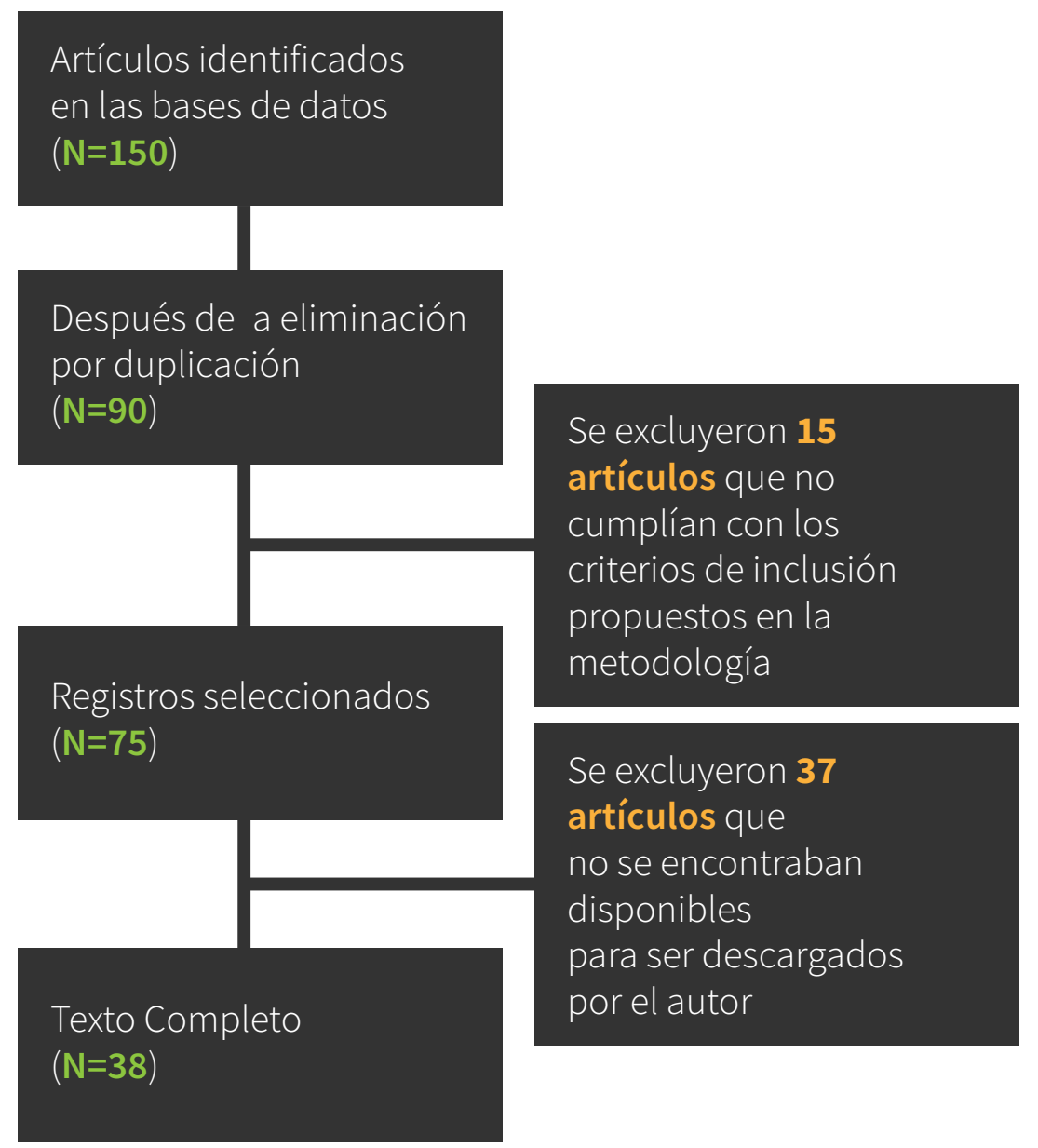

Gráfico 1 Flujograma de extracción de la evidencia

Fuente: Elaboración propia

Tabla 1 Tipos de estudio analizados

\begin{tabular}{|cc|}
\hline Tipo de estudio & $\#$ \\
\hline Revisión de literatura & 7 \\
\hline Revisión sistemática & 6 \\
\hline Estudio piloto & 6 \\
\hline Estudio de caso y control & 7 \\
\hline Estudio prospectivo & 11 \\
\hline ECA & 1 \\
\hline & Total \\
\hline
\end{tabular}

Se pudo identificar literatura sobre la intervención fisioterapéutica en perrosygatos con enfermedades osteomusculares y neuromusculares; los efectos de estas enfermedades se caracterizan por alteraciones en la movilidad, fuerza, locomoción y dolor. Se dividió las categorías de intervención fisioterapéutica en animales en:

\section{Agentes biofísicos}

Se ha reportado el uso de agentes físicos para el tratamiento de lesiones agudas en gatos y perros a través de modalidades terapéuticas como:

$\rightarrow$ Crioterapia y termoterapia. La prescripción se realiza a través de paquetes colocados en la zona de lesión por un período de $\mathbf{5 - 1 0}$ minutos. (Hanks, Levine, \& Bockstahler, 2015; Michel \& Brown, 2011; Millis \& Ciuperca, 2015)

$\rightarrow$ Ultrasonido terapéutico. Modalidad que tiene dos formas de prescripción. La primera con una intensidad de $\mathbf{3 . 3} \mathbf{M H z}$ con una profundidad de $\mathbf{1 . 0}$ $\mathbf{3 . 0} \mathbf{c m}$ y la segunda a $\mathbf{1 . 0} \mathbf{M h z}$ con una profundidad de $\mathbf{2} \mathbf{0}-\mathbf{5 . 0} \mathbf{c m}$, el tiempo de aplicación se recomienda 
12 minutos. La intensidad en general se maneja de $\mathbf{0}$ a $\mathbf{2 . 5 \mathbf { W }} / \mathbf{c m}^{\mathbf{2}}$, el modo se usa continuo al $\mathbf{1 0 0 \%} \mathrm{y}$ pulsado al $\mathbf{2 0 \%}$, el uso de electroestimulación es de $\mathbf{5 0 - 1 5 0 H z}$ con una duración de pulso de $\mathbf{5 0} \mathrm{\mu s}^{1}$. (Hanks, Levine, \& Bockstahler, 2015; Michel \& Brown, 2011; Millis \& Ciuperca, 2015)

El uso de estos agentes físicos ayuda al manejo de dolor, disminución del edema, aumento del rango movimiento y reducción de la espasticidad. (Hanks, Levine, \& Bockstahler, 2015; Michel \& Brown, 2011; Millis \& Ciuperca, 2015)

\section{Ejercicio terapéutico}

El cartílago, músculo, tendón, ligamentosy huesos sufren atrofia, esta causa disminución de movimiento de una extremidad después de una lesión. Estos trastornos musculoesqueléticos son intervenidos a través de ejercicios terapéuticos enfocados en la recuperación de la movilidad y aumento de la fuerza muscular.

$\rightarrow$ Movilizaciones activas y pasivas para fortalecer los tejidos durante el proceso de rehabilitación. (Blackwood, 2013; Mich, 2014; Sharp, 2012a)

$\rightarrow$ La hidroterapia en un método de ejercicio terapéutico enfocado en la recuperación de la movilidad y la fuerza utilizando el agua como agente externo de recuperación. (Blackwood, 2013; Mich, 2014; Sharp, 2012a; 2012b)

$\rightarrow$ Se utiliza la facilitación neuromuscular propioceptiva para aumentar el rango de movimiento y fuerza muscular. (Mich, 2014; Sharp, 2012a; 2012b)

$\rightarrow$ Se utilizan ejercicios de balance, equilibrio y propiocepción en bases inestables y sobre balón terapéutico. (Blackwood, 2013; Mich, 2014; Sharp, 2012a; 2012b)

$\rightarrow$ Aplicación de terapia manual para el manejo de dolor y alteraciones osteomusculares. (Blackwood, 2013; Mich, 2014; Sharp, 2012a; 2012b)

El ejercicio terapéutico es utilizado en patologías como enfermedades de la medula espinal, neuromusculares e intracraneales y lesiones osteomusculares como osteoartritis, fracturas y luxaciones. (Blackwood, 2013; Mich, 2014; Sharp, 2012a; 2012b)

\section{Órtesis, aparatos de soporte y protección}

El fisioterapeuta es el encargado del diseño, adaptación y prescripción de órtesis para animales que presenten problemas para la movilidad y comprometan el desarrollo de sus actividades. Por ejemplo, se han diseñado dispositivos para asistencia en la marcha.

\section{Examinación}

Se identificó el examen de marcha como una herramienta fundamental para determinar alteraciones en la funcionalidad de los animales. El examen se enfoca en patrones de movimiento cinéticos y cinemáticos. (Drum, 2010; Marcellin Little, Drum, Levine, \& McDonald, 2015; Pryor
\& Millis, 2015; Sharp, 2012b)

Se determinó que la rehabilitación en perros y gatos se enfoca en la atención de síntomas como inflamación, edema, dolor, disminución del rango de movimiento, debilidad muscular, rigidez después de una operación, alteraciones vestibulares. (Bockstahler, y otros, 2012; Brown, Boston, Coyne, \& Farrar, 2007; Foss, da Costa, Rajala-Schuttz, \& Allen, 2013; Gordon-Evans, y otros, 2009; Headrick, y otros, 2014; Hyytiäinen, Mölsä, Junnila, Laitinen-Vapaavuori, \& Hielm-Björkman, 2012; Jaeger, Marcellin Little, DePuy, \& Lascelles, 2007; Swimmer \& Rozanski, 2011

Tabla 2 Patologías y modalidades de intervención

\begin{tabular}{|cl}
\hline \multicolumn{1}{c}{ Patologías } & \multicolumn{1}{c}{ Intervención fisioterapéutica } \\
\hline Lesiones medulares & - Ejercicio terapéutico \\
& - Ejercicio funcional \\
Patologías osteomusculares & - Hidroterapia \\
& - Agentes físicos \\
Patologías neurodegenerativas & - Facilitación neuromuscular propioceptiva \\
& - Ejercicios de balance y equilibrio \\
&
\end{tabular}

\section{Discusión}

Como se puede apreciar en los resultados presentados, la fisioterapia en población animal doméstica está tomando cada día más fuerza. A nivel investigativo la rama de la medicina veterinaria ha enfatizado en la participación del área de fisioterapia en procesos de atención a animales con patologías osteomusculares y neuromusculares.

Se evidencia que el tratamiento de rehabilitación en pacientes veterinarios tiene índices significativos en índoles como: función, dolor y actividades de la vida diaria. La rehabilitación en perros y gatos está indicada en procesos agudos y crónicos como patologías osteomusculares y neuromusculares.

Principalmente esta rehabilitación se caracteriza por: ejercicio terapéutico, agentes físicos, ejercicios funcionales y terapia manual las cuales muestran tener un alto nivel de efectividad en este tipo de patologías. (Feeney, y otros, 2007; Levine, Marcellin Little, Millis, Tragauer, \& Osborne, 2010; Mueller, Bockstahler, Skalicky, Mlacnik, \& Lorinson, 2007; Nganvongpanit, Kongsawasdi, Chuatrakoon, \& Yano, 2011; Sullivan, Gordon Evans, Knap, \& Evans, 2013; Waining, Young, \& Williams, 2011)

Los procesos de rehabilitación en perros y gatos han demostrado índices de confiabilidad y validez altos en cuanto a la recuperación funcional de esta población. Por ejemplo, se ha visto un aumento en la capacidad de locomoción de perros con alteraciones neuromusculares que después de sesiones de fisioterapia aumentan su capacidad para realizar el patrón de marcha de manera más efectiva.

Los dueños de estos animales reportan una mejoría en la calidad de vida de estos animales. La fisioterapia en población animal es un área en crecimiento. Aunque existe información, los autores resaltan que se necesitan hacer más investigaciones con el fin de ampliar y enriquecer el conocimiento con respecto al tema a nivel mundial. (Crook, McGowan, \& Pead, 2007; Gaiad, Araujo, Serrão, Miglino, \& Ambrósio, 2014; Gruenenfelder, Boos, Mouwen, \& Steffen, 2006; Challande Kathmann, Cizinauskas, Doherr, Steffen, \& Jaggy, 2006; Levine, Marcellin Little, Millis, Tragauer, \& Osborne, 2010; Marsolais, Dvorak, \& Conzemius, 2002) 


\section{Conclusiones}

La fisioterapia en animales está indicada para procesos agudos y crónicos como patologías osteomusculares, ortopédicas y neuromusculares. La intervención fisioterapéutica se caracteriza por incluir elementos como: ejercicio terapéutico, agentes biofísicos, ejercicios funcionales y terapia manual las cuales muestran tener un alto nivel de efectividad en la recuperación de esta población.

\section{Limitaciones y caminos futuros}

El área de investigación de fisioterapia en animales está en apogeo, se deben seguir realizando estudios en el área con el fin de generar conocimiento en este campo investigativo por parte de fisioterapeutas.

\section{Conflicto de Interés}

Los autores declaran que no existen conflictos de interés.

\section{Referencias}

Blackwood, L. (2013). Cats with Cancer: Where to start. Journal of Feline Medicine and Surgery, 15(5), 366-377. doi:10.1177/1098612X13483235

Bockstahler, B. A., Prickler, B., Lewy, E., Holler, P. J., Vobornik, A., \& Peham, C. (2012). Hind limb kinematics during therapeutic exercises in dogs with osteoarthritis of the hip joints. AJVR American Journal of Veterinary Research, 73(9), 1371-1376. doi:10.2460/ajvr.73.9.1371

Brown, D. C., Boston, R. C., Coyne, J. C., \& Farrar, J. T. (2007). Development and psychometric testing of an instrument designed to measure chronic pain in dogs with osteoarthritis. AJVR American Journal of Veterinary Research, 68(6), 631-637. doi:10.2460/ajvr.68.6.631

Challande Kathmann, I., Cizinauskas, S., Doherr, M. G., Steffen, F., \& Jaggy, A (2006). Daily controlled physiotherapy increases survival time in dogs with suspected degenerative myelopathy. Journal of Veterinary Internal Medicine, 20(4), 927-932. doi:10.1111/j.1939-1676.2006.tb01807.x

Challande Kathmann, I., Demierre, S., \& Jaggy, A. (2001). Rehabilitationsmassnahmen in der Kleintierneurologie [Rehabilitation methods in small animal neurology]. SAT Schweizer Archiv für Tierheilkunde (SCHWEIZ ARCH TIERH), 143(10), 495-502. doi: http://doi.org/10.5169/seals-592855

Crook, T., McGowan, C. M., \& Pead, M. (2007). Effect of passive stretching on the range of motion of osteoarthritic joints in 10 labrador retrievers. VetRecord(160), 545-547. doi: http://dx.doi.org/10.1136/vr.160.16.545

Drum, M. G. (2010). Physical rehabilitation of the canine neurologic patient. Veterinary Clinics of North America: Small Animal Practice, 40(1), 181-193. doi:10.1016/j.cvsm.2009.09.009

Drum, M. G., Bockstahler, B., Levine, D., \& Marcellin Little, D. J. (2015). Feline Rehabilitation. Veterinary Clinics of North America: Small Animal Practice, 45(1), 185-201. doi:10.1016/j.cvsm.2014.09.010

Feeney, L. C., Lin, C.-F., Marcellin Little, D. J., Tate, A. R., Queen, R. M., \& Yu, B. (2007). Validation of two-dimensional kinematic analysis of walk and sit-tostand motions in dogs. AJVR American Journal of Veterinary Research, 68(3), 277-282. doi:10.2460/ajvr.68.3.277

Foss, K. D., da Costa, R. C., Rajala-Schuttz, P. J., \& Allen, M. J. (2013). Force plate gait analysis in Doberman Pinschers with and without cervical spondylomyelopathy. Journal of Veterinary Internal Medicine, 27(1), 106111. doi:10.1111/jvim.12025
Gaiad, T. P., Araujo, K. P., Serrão, J. C., Miglino, M. A., \& Ambrósio, C. E. (2014). Motor Physical Therapy Affects Muscle Collagen Type I and Decreases Gait Speed in Dystrophin-Deficient Dogs. (J. Huard, Ed.) PLoS ONE, 9(4), 9 [e93500]. doi:10.1371/journal.pone.0093500

Gordon-Evans, W. J., Evans, R. B., Knap, K. E., Hildreth, J. M., Pinel, C. B., Imhoff, D. J., \& Conzemius, M. G. (2009). Characterization of spatiotemporal gait characteristics in clinically normal dogs and dogs with spinal cord disease. AJVR American Journal of Veterinary Research, 70(12), 1444 1449. doi:10.2460/ajvr.70.12.1444

Gruenenfelder, F. I., Boos, A., Mouwen, M., \& Steffen, F. (2006). Evaluation of the anatomic effect of physical therapy exercises for mobilization of lumbar spinal nerves and the dura mater in dogs. AJVR American Journal of Veterinary Research, 67(10), 1773-1779. doi:10.2460/ajvr.67.10.1773

Hanks, J., Levine, D., \& Bockstahler, B. A. (2015). Physical agent modalities in physical therapy and rehabilitation of small animals. Veterinary Clinics of North America: Small Animal Practice, 45(1), 29-44. doi:10.1016/j cvsm.2014.09.002

Headrick, J. F., Zhang, S., Millard, R. P., Rohrbach, B. W., Weigel, J. P., \& Millis, D. L. (2014). Use of an inverse dynamics method to compare the threedimensional motion of the pelvic limb among clinically normal dogs and dogs with cranial cruciate ligament-deficient stifle joints following tibial plateau leveling osteotomy or lateral fabellar-tibia. AJVR American Journal of Veterinary Research, 75(6), 554-564. doi:10.2460/ajvr.75.6.554

Henderson, A. L., Latimer, C., \& Millis, D. L. (2015). Rehabilitation and physical therapy for selected orthopedic conditions in veterinary patients. Veterinary Clinics of North America: Small Animal Practice, 45(1), 91-121. doi:10.1016/j.cvsm.2014.09.006

Hyytiäinen, H. K., Mölsä, S. H., Junnila, J. T., Laitinen-Vapaavuori, O. M., \& HielmBjörkman, A. K. (2012). Use of bathroom scales in measuring asymmetry of hindlimb static weight bearing in dogs with osteoarthritis. VCOT Veterinary and Comparative Orthopaedics and Traumatology, 25(5), 390 396. doi:10.3415/VCOT-11-09-0135

Jaeger, G. H., Marcellin Little, D. J., DePuy, V., \& Lascelles, B. D. (2007). Validity of goniometric joint measurements in cats. American Journal of Veterinary Research, 68(8), 822-826. doi:10.2460/ajvr.68.8.822

Levine, D., Marcellin Little, D. J., Millis, D. L., Tragauer, V., \& Osborne, J. A. (2010). Effects of partial immersion in water on vertical ground reaction forces and weight distribution in dogs. AJVR American Journal of Veterinary Research, 71(12), 1413-1416. doi:10.2460/ajvr.71.12.1413

Marcellin Little, D. J., Doyle, N. D., \& Pyke, J. F. (2015). Physical rehabilitation after total joint arthroplasty in companion animals. Veterinary Clinics of North America: Small Animal Practice, 45(1), 145-165. doi:10.1016/j.cvsm.2014.09.008

Marcellin Little, D. J., Drum, M. G., Levine, D., \& McDonald, S. S. (2015). Orthoses and exoprostheses for companion animals. Veterinary Clinics of North America: Small Animal Practice, 45(1), 167-183. doi:10.1016/j.cvsm.2014.09.009

Marsolais, G. S., Dvorak, G., \& Conzemius, M. G. (2002). Effects of postoperative rehabilitation on limb function after cranial cruciate ligament repair in dogs. AVMA Journal of the American Veterinary Medical Association, 220(9), 1325-1330. doi:10.2460/javma.2002.220.1325

Mich, P. M. (2014). The Emerging Role of Veterinary Orthotics and Prosthetics (V-OP) in Small Animal Rehabilitation and Pain Management. Topics in Companion Animal Medicina, 29(1), 10-19. doi:10.1053/j.tcam.2014.04.002

Michel, K. E., \& Brown, D. C. (2011). Determination and application of cut points for accelerometer-based activity counts of activities with differing intensity in pet dogs. AJVR American Journal of Veterinary Research, 72(7), 866870. doi:10.2460/ajvr.72.7.866

Millis, D. L., \& Ciuperca, I. A. (2015). Evidence for Canine Rehabilitation and Physica Therapy. Veterinary Clinics of North America: Small Animal Practice, 45(1), 1-27. doi:10.1016/j.cvsm.2014.09.001

Mueller, M., Bockstahler, B. A., Skalicky, M., Mlacnik, E., \& Lorinson, D. (2007). Effects of radial shockwave therapy on the limb function of dogs with hip osteoarthritis. VetRecord(160), 762-765. doi:10.1136/vr.160.22.762

Nganvongpanit, K., Kongsawasdi, S., Chuatrakoon, B., \& Yano, T. (2011). Heart Rate Change during Aquatic Exercise in Small, Medium and Large Healthy Dogs. Thai Journal of Veterinary Medicine, 41(4), 455-462. Retrieved from https://www.tci-thaijo.org/index.php/tjvm/article/view/9621 
Nolen, R. S. (2009). Pet rehab becoming mainstream practice: physical therapy for animals increasingly seen as viable treatment option, 20090915T. (JAVMA Journal of the American Veterinary Medical Association) Retrieved from JAVMA news, Companion animals: https://www.avma.org/News/ JAVMANews/Pages/091001c.aspx

Pryor, B., \& Millis, D. L. (2015). Therapeutic Laser in Veterinary Medicine. Veterinary Clinics of North America: Small Animal Practice, 45(1), 45-56. doi:10.1016/j.cvsm.2014.09.003

Sharp, B. (2012a). Feline Physiotherapy and Rehabilitation: 1. Principles and potential. Journal of Feline Medicine and Surgery, 14(9), 622-632. doi:10.1177/1098612X12458209

Sharp, B. (2012b). Feline Physiotherapy and Rehabilitation: 2. Clinical application. Journal of Feline Medicine and Surgery, 14(9), 633-645. doi:10.1177/1098612X12458210

Sims, C., Waldron, R., \& Marcellin Little, D. J. (2015). Rehabilitation and Physical Therapy for the Neurologic Veterinary Patient. Veterinary Clinics of North America: Small Animal Practice, 45(1), 123-143. doi:10.1016/j.cvsm.2014.09.007

Sullivan, M. O., Gordon Evans, W. J., Knap, K. E., \& Evans, R. B. (2013). Randomized, controlled clinical trial evaluating the efficacy of pulsed signal therapy in dogs with osteoarthritis. Veterinary Surgery, 42(3), 250-254. doi:10.1111/j.1532-950X.2012.01066.X

Swimmer, R. A., \& Rozanski, E. A. (2011). Evaluation of the 6 minute walk test (6MWT) in pet dogs. Journal of Veterinary Internal Medicine, 25(2), 405406. doi:10.1111\%2Fj.1939-1676.2011.0689.x

Waining, M., Young, I. S., \& Williams, S. B. (2011). Evaluation of the status of canine hydrotherapy in the UK. VetRecord(168), 407. doi:10.1136/Vr.c6842 